REGARDS

SUR L'ECONOMIE ALLEMANDE

BULLETIN ECONOMIQUE DU CRAC
Regards sur l'économie allemande

Bulletin économique du CIRAC

$71 \mid 2005$

Varia

\title{
Directive 'Services' : les enjeux du débat allemand
}

Isabelle Bourgeois

\section{OpenEdition}

\section{Journals}

Édition électronique

URL : http://journals.openedition.org/rea/322

DOI : $10.4000 /$ rea. 322

ISBN : 978-2-8218-0838-6

ISSN : 1965-0787

Éditeur

CIRAC

Édition imprimée

Date de publication : 1 mai 2005

Pagination : 5-14

ISSN : 1156-8992

Référence électronique

Isabelle Bourgeois, "Directive 'Services' : les enjeux du débat allemand ", Regards sur l'économie

allemande [En ligne], 71 | mai 2005, document 1, mis en ligne le 06 mai 2008, consulté le 22 avril 2019. URL : http://journals.openedition.org/rea/322 ; DOI : 10.4000/rea.322 


\section{Directive 'Services' : les enjeux du débat allemand}

\section{Isabelle Bourgeois}

«La directive, dans sa version actuelle, ne débouchera assurément pas sur une loi ", déclarait officiellement le chancelier Schröder à l'issue du Conseil européen de Bruxelles, le 23 mars 2005. Et, soulignant la profonde entente entre la France et l'Allemagne sur le sujet, il expliquait: "la liberté des services au sein du marché communautaire nous est indispensable, mais il nous faut aussi préserver le modèle social européen ; nous ne voulons donc pas de dumping salarial, pas de dumping social ».

L'opposition du chancelier au projet de directive "relative aux services dans le marché intérieur " est soudaine. Le 26 octobre 2004 encore, dans le quotidien économique Handelsblatt, Gerhard Schröder développait sa vision d'un programme d'action pour l'Europe dans le cadre de la stratégie de Lisbonne, intitulé « 7 opportunités pour plus de croissance en Europe ». La quatrième portait sur " l'énorme potentiel de croissance " inhérent à " un véritable marché intérieur des services..., bénéfique à des millions d'entreprises rien qu'en Allemagne ». Et il considérait alors le projet de directive comme "la démarche adaptée en toute logique pour atteindre ces objectifs ». En effet, poursuivait-il, « la directive 'services' crée un cadre permettant aux entreprises européennes de développer leur compétitivité et de créer plus d'emplois ».

En quelques mois, le gouvernement fédéral a effectué un revirement total. Alors que s'ouvre la campagne pour les élections régionales en Rhénanie du NordWestphalie (22 mai), suivie de diverses échéances qui culmineront avec les élections au Bundestag à l'automne 2006, l'argument de la dynamique de croissance attendue a cédé le pas à la focalisation du discours politique sur l'immigration de travailleurs est-européens offrant leurs prestations au noir. S'emparant des positions des détracteurs du projet, le gouvernement de coalition et le parti SPD ont lancé en avril 2005 une offensive contre le "dumping salarial »et prônent désormais l'adoption d'un salaire minimum légal comme la généralisation des dispositions sur les travailleurs détachés adoptées en 1996 dans le BTP. Opposition, fédérations professionnelles, experts et syndicats se livrent à une surenchère d'arguments n'ayant plus que des liens éloignés avec un texte soumis actuellement à la procédure du débat parlementaire européen.

Pour le dire d'entrée : en réalité - par-delà les propos tenus actuellement dans l'espace public -, la position de l'Allemagne quant à l'achèvement du marché intérieur, c'est-à-dire à la mise en œuvre du principe de la libre circulation des services, après celle des biens et des personnes, n'a nullement varié. Pour le gouvernement comme l'opposition, pour les milieux patronaux comme pour les syndicats, l'achèvement du marché intérieur reste une priorité. L'intégration dans l'UE est en effet indissociable de la prospérité de l'économie allemande depuis l'adoption du Traité de Rome et du principe de libre circulation au fondement de l'espace communautaire : nation exportatrice par excellence, l'Allemagne réalise, bon an, mal an, plus de la moitié de ses ventes de biens dans I'UE 15, et près des trois quarts dans l'UE 25. Elle a réitéré son engagement dans la poursuite du processus d'achèvement du marché intérieur à l'échelon communautaire lors du Sommet européen de Lisbonne en 2000, à l'échelon

Libre circulation des services : une priorité pour l'Allemagne 
national en lançant notamment les réformes structurelles de l'Agenda 2010. Le projet de directive 'Services' du 13-01-2004 - que personne ou presque, outreRhin, n'appelle « directive Bolkestein » - s'inscrit expressément dans cet objectif dit « stratégie de Lisbonne », et qui vise à « faire de l'UE, à l'horizon 2010, l'économie de la connaissance la plus compétitive et la plus dynamique du monde $»$.

\section{Les obstacles identifiés en juillet 2002 par le \\ Rapport de la Commission sur l'état du marché intérieur des services [COM(2002) 441 final]}

II est difficile de chiffrer précisément le poids des services dans la structure de l'économie allemande ou européenne, étant donné que le secteur est encore très mal connu des statistiques qui commencent seulement à tenter d'en définir le périmètre - un exercice d'autant plus délicat que les différentes activités de services se caractérisent par une forte interdépendance. C'est donc pour une approche délibérément "horizontale », et non sectorielle, qu'avait opté la Commission pour effectuer son diagnostic des dysfonctionnements constatés à toutes les étapes de la prestation de services : obstacles à l'établissement du prestataire dans un autre Etat membre, difficultés rencontrées pour l'utilisation des inputs nécessaires à la fourniture des services transfrontaliers (matériels, salariés, etc.), restrictions à la promotion, à la distribution (vente, après-vente...), difficulté d'accès, pour les consommateurs, à l'information sur les prestations, aux prestations elles-mêmes, et de ce fait, faible protection contre les comportements abusifs.

L'analyse de la nature de ces obstacles, que le rapport dénomme des «frontières » conformément à la définition du marché intérieur du Traité CE ("un espace sans frontières intérieures »), révèle des points communs transversaux, ou " horizontaux », identifiables par-delà la diversité des situations concrètes. Le principal est que «les Etats membres appliquent un régime unique tant aux prestataires de services qui veulent s'établir sur leur territoire qu'à ceux qui veulent prester leurs services depuis leur pays d'origine. Pour ces derniers, qui sont déjà soumis aux règles et au contrôle de leur pays d'établissement, il peut en résulter une duplication des règles et des contraintes disproportionnées ". C'est ce constat qui légitimera, dans le projet de directive 'Services', l'extension au secteur du « principe du pays d'origine » tel qu'il prévaut dans l'organisation du marché intérieur des biens et produits depuis l'arrêt «Cassis de Dijon » rendu par la Cour européenne de justice en 1979. Le second point commun est "l'incertitude juridique qui découle de l'application, au cas par cas, par les autorités nationales, d'exigences peu claires dont le résultat est souvent imprévisible ».

II en découle que ces frontières "reviennent très cher aux entreprises engagées dans les activités entre Etats membres ", essentiellement aux PME ; elles sont majoritaires dans le secteur de l'offre de services. C'est donc là que réside l'un des principaux handicaps à l'internationalisation (ou plutôt européanisation) des PME, et donc au développement de leur compétitivité - un autre des axes majeurs de la stratégie de Lisbonne. Mais ce sont plus encore les utilisateurs de services, « et en particulier les consommateurs qui paient le prix de ces restrictions en n'étant pas en mesure de profiter d'une plus grande variété de services, de meilleure qualité et à un prix plus compétitif ». Finalement, poursuit le rapport, "cette situation a également un effet sur leur qualité de vie » : "les citoyens européens souffrent du manque à gagner en termes de créations d'emplois dans l'ensemble du secteur des services ».

Ces frontières sont de deux ordres : soit elles « découlent directement et indirectement de contraintes juridiques », soit elles "découlent de facteurs non juridiques ». Elles s'appliquent à toutes les étapes liées à la prestation de service.

- La première catégorie comprend principalement : la limitation de l'accès à certaines activités, les exigences de résidence ou de nationalité, les procédures d'autorisation ou de déclaration préalables, les exigences de qualification professionnelle (pas seulement dans le cas des professions réglementées), ou encore les règles et pratiques en matière de fixation des prix, de facturation et de paiement, la fiscalité (dont la TVA), de même que la diversité des régimes de protection sociale.

- La seconde catégorie comprend les difficultés liées au manque d'information sur l'environnement réglementaire respectif ou la méconnaissance des principes du marché intérieur ou les difficultés d'ordre culturel ou linguistique (pratiques administratives ou habitudes de consommation). Mais le principal obstacle est celui-ci : "les entreprises pensent encore 'national' ».

La liste des «frontières » est longue et, plus important : elle reflète, à y bien regarder, l'énorme diversité des règles et pratiques nationales liées au fonctionnement de l'ensemble des activités au sein de l'espace communautaire. Les services sont non seulement interdépendants avec les activités de production industrielle, mais ils sont également interdépendants entre eux : via l'embauche d'un salarié, par exemple, une prestation fait appel en amont aux services de formation, mais aussi à l'ensemble des services liés à la protection sociale de ce salarié, sans parler des tâches effectuées par ce salarié et les services auxquels il recourt pour ce faire. Les disparités s'expliquent par la genèse historique des systèmes nationaux, mais proviennent aussi très largement d'une transcription inadéquate de la réglementation européenne. Les frontières ont en effet pour point commun d'être évolutives et horizontales. Cela est particulièrement flagrant dans le fait que « l'Etat membre de destination traite le prestataire de services comme s'il s'agissait d'une entreprise établie sur son territoire et le soumet donc pleinement à son régime juridique »- une pratique en franche contradiction avec la jurisprudence de la Cour européenne de justice, comme le souligne le rapport. II établit enfin l'origine commune de ces frontières, dans "le manque de confiance mutuelle entre les Etats membres » qui se traduit généralement par «la protection d'intérêts économiques nationaux ». 
L'Allemagne a tout à gagner de la "levée des obstacles qui entravent le développement des activités de services dans le Marché intérieur ", comme le formule le projet de directive. Car les services, un secteur pourtant dynamique et qui occupe quelque $70 \%$ des salariés dans tous les Etats membres, restent encore largement confinés dans les frontières nationales. Si cela s'explique pour certains d'entre eux par le fait qu'ils sont liés aux activités de proximité ou tributaires du contexte culturel, la majorité des services se heurte à des barrières principalement d'ordre réglementaire ou administratif qui complexifient considérablement l'offre de prestations hors du pays d'origine et interdisent aux consommateurs (particuliers ou entreprises) des autres Etats membres de recourir au prestataire de leur choix. Dans un rapport remis au Conseil et au Parlement européen en juillet 2002 (voir l'encadré ci-dessus), la Commission avait dressé, à l'issue d'un long processus de consultation, un état des lieux détaillé des "difficultés concernant les activités de services entre les Etats membres". Achevant la première phase de la "mise en œuvre de la stratégie pour le Marché intérieur des services ", ce diagnostic avait alors débouché sur l'adoption du projet de directive 'Services' aujourd'hui soumis au vote du Parlement européen.

Dans le cas de l'Allemagne, les difficultés et obstacles identifiés dans le cadre de cette procédure se révèlent un lourd handicap pour une industrie non seulement fortement internationalisée, mais qui a aussi pour seconde caractéristique d'être une 'industrie de services' (voir REA 58/02). Les premières enquêtes et études publiées par Destatis et la fédération des Chambres de commerce et d'industrie DIHK depuis 2002 laissent entrevoir outre-Rhin une étroite interdépendance entre le secteur manufacturier et le tertiaire. Des liens quasi symbiotiques se sont constitués au fil des mutations de la décennie écoulée, traduisant notamment la globalisation des activités depuis la rapide diffusion des TIC, et se manifestant par une double tendance : à l'externalisation des fonctions tertiaires par les entreprises industrielles et à la création, à proximité des gisements de commandes, de nouveaux prestataires (du transport au contrôle de gestion en passant par le traitement des déchets). Ces liens se reflètent à l'export, l'industrie allemande commercialisant à la fois un produit et les services afférents : installation, service après-vente, maintenance, entretien, formation, assurances, etc. Ces prestations qui accompagnent le produit industriel et contribuent à la réputation mondiale du made in Germany (mais qui sont encore généralement comptabilisées en termes statistiques parmi les activités du secteur manufacturier) représentaient quelque $60 \%$ des activités des industriels allemands en 2002, selon une enquête de la DIHK. Selon la première enquête sur les services liés aux produits menée par Destatis en 2002, plus des trois quarts $(77 \%)$ des entreprises tertiaires interrogées offraient des prestations de ce type.

Plus révélatrice encore est la première enquête menée par Destatis en 2003 (les premières conclusions en ont été publiées en avril 2005) sur la demande de services émanant de sept secteurs : industrie, BTP, commercialisation et entretien de biens de consommation (dont automobile), hôtellerie/gastronomie, transports et communication, location de biens mobiliers, ainsi que de la catégorie incluant le traitement de l'eau et des déchets comme le cinéma et l'audiovisuel. Jusqu'ici, seule l'offre était prise en compte. Or selon Destatis, la demande émanant des entreprises interrogées a atteint un volume de 423 milliards $€$ (pour un CA total de 2974 milliards $€$ ). Sur ce montant, 16 milliards $€$ étaient consacrés à l'investissement : $28 \%$ aux TIC, $24 \%$ à l'achat de droits d'exploitation, $17 \%$ à la R\&D, $10 \%$ au marketing et $21 \%$ à d'autres services. Les 407 milliards $€$ restants étaient destinés surtout à la consommation de prestations. La demande la plus forte a émané de l'industrie (40\%), suivie de loin par le secteur transports et communications (24\%), le commerce (18\%), les prestataires de services aux entreprises (11\%) ; le BTP, la gastronomie/hôtellerie et les services publics se sont partagé le reste $(7 \%)$.
Lever les obstacles à la croissance et l'emploi dans les services

RFA : des handicaps surtout pour les services aux entreprises

Une forte demande de services dans l'industrie allemande 
$1 \%$ seulement des entreprises achètent des services hors de RFA

IDE et fusions comme succédané à un marché intérieur des services qui n'existe pas encore

Unanimité sur la nécessité de parachever le marché intérieur...
Si la demande vaut pour toutes les fonctions (sauf la direction), certaines prestations sont plus systématiquement achetées que d'autres (par plus de $80 \%$ des entreprises) : le conseil juridique, fiscal et l'assurance. Pour ces activités, de même que pour le leasing, les baux ou les prestations financières, la majorité des entreprises $(90 \%)$ est fidèle à son prestataire. A l'inverse, plus d'un quart d'entre elles fait jouer la concurrence en ce qui concerne les études de marché, la publicité, la GRH ou les services d'ingénierie. La fidélité des entreprises s'accompagne également d'un fort ancrage régional des liens avec le prestataire, ce qui confirme la tendance observée par la DIHK à la constitution de grappes d'activités. $1 \%$ seulement des entreprises affirme recourir à un prestataire hors du territoire national ; le critère déterminant pour le choix est en effet la proximité. Le premier des handicaps à la conclusion d'un contrat avec un prestataire étranger est dès lors la distance géographique. Ce n'est qu'ensuite que viennent les barrières légales ou administratives (fondamentales en matière de conseil juridique et fiscal), le manque de confiance (assurance et services financiers) ou les obstacles linguistiques (GRH). Par contre, le coût (même élevé) des prestations n'est pas considéré comme un handicap.

Voilà qui confirme, à l'échelle d'un segment des services dans un Etat membre, certaines des grandes tendances esquissées par Eurostat à l'échelon de l'UE et rappelées par le rapport de la Commission sur les obstacles au marché intérieur des services. "Même si les statistiques sur l'industrie sont probablement surestimées du fait de l'intégration des services dans la production, ... le poids des services dans les échanges est sans rapport avec leur poids dans l'économie : les services ne représentent encore que 21,6\% de l'ensemble des transactions commerciales de l'Union européenne en 1999, dont 11,9 \% à l'intérieur de l'UE 15 ». Cette réalité se traduit par le fait que «les acteurs ont tendance à avoir recours à l'investissement direct à l'étranger et aux fusions plutôt qu'aux échanges directs ». Voilà qui contribue certainement à expliquer l'importance des IDE allemandes à l'étranger. La faiblesse des échanges en matière de services serait ainsi un motif pour la délocalisation des activités, notamment vers les PECO. Mais ces pratiques, adoptées en quelque sorte à défaut, limitent considérablement le développement des activités hors du territoire national.

La fédération allemande de la construction mécanique (VDMA) qui est l'une des branches phares de l'industrie allemande et sa championne à l'export, songe dès lors à l'énorme potentiel que représente pour sa branche la libre circulation des services : toute machine-outil ou installation industrielle étant vendue avec les services afférents (de l'installation à la formation des équipes en passant par la planification du renouvellement du parc), «nous pourrons à l'avenir vendre plus de machines ", explique-t-on à la VDMA. De son côté, le secteur du conseil aux entreprises estime, via sa Fédération BDU, qu'il est primordial "que les conseillers et auditeurs puissent servir leurs clients hors des frontières nationales promptement et avec facilité ». Dans une proposition conjointe « d'action pour la politique européenne des cinq prochaines années » présentée en décembre 2004 et destinée aux acteurs politiques, les fédérations du patronat (BDA) et de l'industrie (BDI) formulaient une série de recommandations, dont celle-ci : "les acteurs économiques allemands continuent d'accorder la priorité absolue au développement et à l'achèvement du marché intérieur... Mais il s'agit dans le même temps d'éviter une harmonisation démesurée dans les domaines qui ne sont pas décisifs pour le bon fonctionnement du marché intérieur et dans lesquels l'UE n'a pas de compétences explicites ».

Or toutes ces voix se sont fait singulièrement muettes après le Conseil européen de mars dernier. Seule, la Fédération du commerce extérieur (BGA) continue de prôner publiquement une adoption rapide et inconditionnelle de la directive ; mais son président, Anton F. Börner, ne ménage pas sa critique au gouvernement fédéral pour son revirement : "si nous avions abordé à l'époque la question de la libre circulation des biens avec la même frilosité, nous n'aurions pas aujourd'hui ce marché intérieur [i.e. européen] si bénéfique à l'Allemagne ». 


\section{Le projet de Directive relative aux services dans le marché intérieur [COM(2004) 2 final]}

L'objectif de la directive est de créer un cadre juridique qui supprime les « frontières » identifiées par le rapport de 2002 sur l'état du marché intérieur des services ; et ce, aux trois niveaux où se situent les obstacles : liberté d'établissement des prestataires, libre circulation des services et confiance mutuelle entre les Etats membres.

La démarche proposée pour l'achèvement du marché intérieur à l'horizon 2010 - une des conditions pour dynamiser la croissance et l'emploi en Europe - est «dynamique »: il s'agit d'un processus où les diverses étapes de la mise en œuvre s'accompagnent d'une évaluation de l'évolution du marché afin de détecter d'éventuels champs d'action nouveaux. Le projet de directive est lui-même le fruit d'une définition progressive des objectifs à atteindre et d'une série d'ajustements intervenus au fil de son élaboration depuis le Conseil européen de Lisbonne en 2000. Et il s'inscrit à la fois dans la " cohérence avec les autres politiques communautaires » (Exposé des motifs, point 5) et dans la continuité du droit communautaire présent ou futur; par exemple, il " ne préjuge pas d'autres initiatives communautaires, législatives ou non, dans le domaine de la protection des consommateurs » (Exposé des motifs, point 3 a).

La nature du texte est celle d'une directive-cadre, ce qui signifie qu'il " n'a pas pour objet de fixer des règles détaillées ou d'harmoniser la totalité des règles des Etats membres applicables aux activités de services " (ibid.). L'expérience a en effet démontré qu'une démarche d'harmonisation aurait comporté le risque d'aboutir à une " sur-réglementation et à l'uniformisation " des spécificités nationales, a fortiori dans un domaine aussi complexe et empreint de diversité que celui des services. Dans son approche, la directive respecte ainsi pleinement le principe de subsidiarité au fondement de l'organisation de l'Europe.

Plus pragmatiquement, une harmonisation de l'ensemble des systèmes nationaux régulant les services serait impossible. Non seulement l'étendue des champs concernés par l'acte de prester un service (fixation des prix, régimes fiscaux, pratiques salariales, systèmes de formation ou de protection sociale...) ne le permet pas, mais le principe de subsidiarité l'interdit pour un certain nombre d'entre eux : les questions sociales par exemple relèvent de la pleine souveraineté des Etats membres. Ces domaines, auxquels il faut ajouter l'éducation publique et l'administration (des services non marchands, au cœur du pouvoir régalien de chacun des Etats membres), ou encore les activités culturelles, n'entrent donc pas dans le champ d'application du projet de directive. Divers secteurs connaissant une réglementation communautaire par ailleurs (services financiers, services et réseaux de communications électroniques, transports) sont eux aussi exclus, de même que la fiscalité.

Le projet de directive s'applique donc exclusivement aux services fournis en tant qu'activité économique destinée aux entreprises et aux particuliers, tels que les a définis dans sa jurisprudence la Cour européenne de justice (art. 4, al. 1). Elle avait été saisie notamment pour de nombreuses entraves au principe de libre circulation des services tel qu'il figure au Traité de Rome, ce qui l'avait amenée à définir progressivement les activités de services. Conformément à sa nature de projet de directive-cadre, le texte ne définit donc pas de liste des services concernés (ce serait contraire à son approche); mais il énonce un certain nombre de règles et de principes visant à mettre en œuvre leur libre circulation.

Si le principe de subsidiarité l'amène à ne procéder à aucune " harmonisation détaillée » pour éviter "les interférences avec l'organisation institutionnelle de la régulation des services des Etats membres " (Exposé des motifs, point 6 b), il explique également le recours au principe du pays d'origine qui " permet de réaliser l'objectif d'assurer la libre circulation des services tout en laissant coexister de manière pluraliste les régimes juridiques des Etats membres avec leurs spécificités et particularités, ces dernières ne pouvant être utilisées pour restreindre les services d'un prestataire établi dans un autre Etat membre " (ibid.). Le projet de directive est donc en conformité absolue avec le principe de subsidiarité. Concrètement, cela signifie par exemple que, puisqu'une entreprise est 'homologuée' (inscription au registre du commerce, preuve des qualifications requises...) dans son pays d'origine, elle n'a plus à le faire dans le pays d'accueil (il reconnaît de jure et de facto cette 'homologation') lorsqu'elle désire y offrir ses services. En revanche, elle doit se soumettre aux normes en vigueur dans le pays d'accueil, donc y respecter entre autres le droit du travail. C'est donc bien le principe de subsidiarité qui sous-tend le recours au principe du pays d'origine - en association avec le principe du pays d'accueil ; la conjugaison des deux permet en effet de mieux respecter les prérogatives de chacun des Etats membres dont les régimes juridiques peuvent ainsi « coexister de manière pluraliste ».

De même, le principe de proportionnalité qui fonde les choix législatifs du texte et vise à "proposer un instrument équilibré dont les dispositions ne vont pas au-delà de ce qui est nécessaire pour réaliser l'objectif " (Exposé des motifs, point $6 \mathrm{c}$ ). C'est ainsi que le texte privilégie les méthodes de régulation que sont la " coopération administrative » et "l'adoption de codes de conduite au niveau communautaire », réservant l'harmonisation aux rares cas où ces deux méthodes se révéleraient insuffisantes (la protection des consommateurs est expressément nommée ici). II s'agit en réalité d'un dosage subtil : "la directive propose un équilibre entre, d'une part, la portée donnée au principe d'origine et, d'autre part, le degré d'harmonisation, de coopération administrative et de renvoi aux codes de conduite ainsi que le champ et le nombre de dérogations au principe du pays d'origine. Le dosage ainsi proposé représente une approche sélective et nuancée qui tient pleinement compte de tous les intérêts concernés 》 (ibid.).

Autrement dit : sur la base de ces quelques principes et choix de méthode, le projet de directive laisse une grande latitude d'action à chacun des Etats membres pour réaliser la libre circulation des services. L'introduction du principe du pays d'origine dans l'ensemble des normes constitue le pivot de cette approche. Autrement dit : le principe de la « confiance mutuelle entre Etats membres » (Résumé, point 5).

Les règles préconisées se résument dès lors à quelques mécanismes simples : établir des obligations d'assistance mutuelle entre autorités nationales, créer des guichets uniques pour simplifier les procédures et démarches administratives des prestataires désireux de s'installer dans le pays d'accueil, garantir la libre circulation des services dans la législation nationale (dont le droit à l'accès aux services), supprimer en conséquence les obstacles réglementaires afférents, et procéder à une harmonisation complémentaire sur certaines questions spécifiques. Quant à leur mise en œuvre, elle obéit à une approche graduelle - et non statique - permettant de " prendre en compte l'évolution de l'intégration communautaire dans d'autres domaines " (Exposé des motifs, point $3 \mathrm{~d}$ ).

Ce projet de directive-cadre se veut donc perfectible, l'achèvement du marché intérieur des services étant un processus de longue haleine s'effectuant de surcroît dans le contexte évolutif du processus plus général de l'intégration économique et sociale de l'UE. 
... mais l'espace public est accaparé par les voix critiques

La « défense du modèle social européen », un argument factice

Crispations corporatistes de l'artisanat allemand

Et craintes pour la compétitivité dans une Europe élargie

\section{Principe du pays d'origine et salaire minimum}

II faut dire que les voix positives ont été mises en minorité outre-Rhin dans l'espace public au fil des derniers mois - un cas d'école illustrant la théorie de la " spirale du silence » développée voici trente ans par E. Noelle-Neumann pour expliquer les processus selon lesquels, dans une société médiatisée, se diffusent dans l'espace public certaines opinions qui finissent par l'emporter sur d'autres et constituent alors, via l'interaction avec le jeu médiatique, un courant considéré comme majoritaire. C'est ainsi qu'aujourd'hui, les voix critiques ont pris le relais, mettant à profit l'attention médiatique (comme les lois propres à l'information), et usant avec facilité de l'amalgame à propos d'un texte somme toute peu connu de l'opinion, et donc malléable à volonté. La campagne électorale qui débute ne contribue pas à la sérénité d'un débat où s'affrontent plus des émotions travesties en idéologie que des arguments factuels.

Les 'arguments' des détracteurs du texte, qui se rejoignent sur le slogan de la " défense du modèle social européen " comme dans le refus du principe du pays d'origine, peuvent se résumer à deux catégories. La première rassemble toutes les crispations corporatistes avivées par l'angoisse de voir lever des statuts ou des régimes protégeant certains marchés ou professions. La seconde peut se définir, en termes de communication stratégique, comme le recours par certaines organisations à des arguments-prétextes diffusés dans l'espace public pour susciter une demande d'action à laquelle seules ces organisations sauront apporter la réponse appropriée. Ces deux catégories, enfin, où 'arguments' exposés et motivations sous-jacentes s'interpénètrent en cascade, nourrissent les discours politiques qui s'affrontent à l'approche des élections. En un mot : l'espace public allemand est actuellement aux prises avec ce qu'il convient d'appeler ainsi une 'spirale de la désinformation' ; nous en présentons ici les principaux éléments.

La plus caractéristique des positions entrant dans la première catégorie est celle de la fédération allemande de l'artisanat qui récuse vivement le principe du pays d'origine : sous couvert de garantir la protection des consommateurs et de lutter contre le risque de "dumping salarial », le secteur cherche en réalité à préserver son organisation statutaire millénaire en corporations dont le pivot est la limitation d'accès aux professions artisanales, concrétisée par l'obligation, faite à quiconque désire créer une entreprise dans l'un des métiers figurant dans la nomenclature du Code de l'artisanat, de disposer d'un diplôme de Meister: de maître-artisan (voir REA 48/00). Or voici deux ans, la liste des métiers ainsi réglementés avait été allégée dans le contexte de la levée de certaines rigidités du marché de l'emploi. Non sans de vives discussions d'ailleurs, et seulement après que le plus ardent défenseur des intérêts du secteur, le ministre-pésident de Bavière, Edmund Stoiber (CSU), aura contribué à faire adopter en contrepartie au Bundesrat des dispositions plus contraignantes pour les chômeurs dans la loi Hartz IV.

Pour illustrer les risques inhérents au principe du pays d'origine, l'exemple le plus répandu dans les médias, et relayé par la sphère politique, dont le chancelier ou le président du parti CSU, a été celui de ces ouvriers polonais, arrivés en Allemagne en " abusant » de la libre circulation des travailleurs indépendants dans l'UE, et embauchés dans les abattoirs allemands pour des salaires largement inférieurs aux normes de branche (il faut préciser ici que l'affaire en question avait donné lieu à une jurisprudence concluant à des circonstances atténuantes, la législation allemande, peu concluante, favorisant ce genre d'abus). Ces salariés se sont trouvés érigés en représentants de tous les Européens de l'Est, suspectés désormais de forcer le "dumping social » et d'accroître le chômage outre-Rhin. Cet exemple, censé démontrer les méfaits d'un marché européen des services mais qui traduit surtout la crainte de certains secteurs de l'économie de perdre leur compétitivité face au dynamisme des nouveaux Etats membres (avec leurs régimes fiscaux et salariaux plus attractifs pour des ni- 
veaux de qualification comparables ou sensiblement comparables), a pris une place telle dans l'espace public que le gouvernement fédéral a choisi d'engager à la mi-avril des négociations avec la Pologne pour limiter ces abus, selon l'explication officielle.

La focalisation sur la Pologne n'est pas le fruit du hasard : dès avant l'élargissement, nombre de ressortissants des Dix avaient créé des micro-entreprises artisanales en Allemagne, principalement des Polonais. Cette tendance s'est accentuée avec l'allègement de l'accès à certains métiers artisanaux (la pose de parquets ou de dallages, par exemple). C'est ainsi que dans le seul Land de Rhénanie du Nord-Westphalie, 900 travailleurs indépendants est-européens (dont $90 \%$ de Polonais) ont déclaré la création de leur entreprise auprès des chambres de l'artisanat: dans les métiers cités, mais aussi dans le travail des métaux, l'horticulture ou la culture de produits maraîchers (la récolte des asperges est un autre exemple célèbre), et dans le transport routier.

De quoi esquisser, innombrables 'exemples' à l'appui, un scénario apocalyptique de démontage du « modèle social européen » (allemand, en l'occurrence). Or ces 'exemples' pêchent à maints égards : d'abord, ils ne peuvent être associés à une directive qui n'est encore qu'à l'état de projet. Ensuite, ils font l'amalgame entre des dysfonctionnements constatés et une lecture peu rigoureuse d'un texte qui ne s'applique dans l'ensemble qu'aux services de nature économique destinés aux entreprises et exclut de son champ d'application un certain nombre de domaines ou leur réserve un traitement particulier. De même, les 'arguments' avancés taisent volontairement une disposition adoptée dans le cadre de l'élargissement de I'UE : la limitation, à titre transitoire, de la libre circulation (établissement) des salariés est-européens jusqu'en 2012. Enfin, et cet aspect est fondamental, ils soulèvent deux questions indépendantes du projet de directive : le problème général du travail illégal d'un côté (voir REA 70/05) ; de l'autre, celui, plus spécifique à l'Allemagne, d'une mauvaise transposition de la Directive 96/71/CE (du 16-12-1996) concernant le détachement de travailleurs effectué dans le cadre d'une prestation de services. L'Allemagne l'avait transposée en mars 1996, avant même son adoption définitive, et limitée pour l'essentiel au secteur du BTP (voir à la fin de ce numéro).

C'est donc aussi la loi allemande sur les travailleurs détachés (ArbeitnehmerEntsendegesetz) qui se trouve en débat. En conformité avec la directive, elle repose elle aussi sur le principe du pays d'accueil, étendant aux secteurs et métiers concernés les normes salariales allemandes (minima de branche). Venue à point nommé alors que la crise du BTP battait son plein dans les nouveaux Länder, elle avait vocation de lutter contre le chômage des salariés estallemands du secteur en les protégeant de la 'concurrence déloyale' de la main d'œuvre est-européenne. Cette protection s'effectue lors du contrôle du versement du salaire minimum allemand (principe du pays d'accueil) aux travailleurs détachés, qui ne prend pas en compte les majorations et suppléments versés par les employeurs du pays d'origine. Considérant cette pratique comme un obstacle à la libre prestation des services (via le renchérissement des coûts salariaux), la commission européenne avait saisi en 2002 la Cour européenne de justice ; celle-ci a rejeté le recours le 14 avril 2005, considérant que la directive $96 / 71 / C E$ ne requiert pas la prise en compte des prestations salariales complémentaires, versées, elles, par l'employeur dans le pays d'origine.

Voilà qui devrait aviver l'actuel débat portant sur l'extension de cette loi à toutes les branches, et donc sur la généralisation de salaires minima pour empêcher le " dumping salarial » d'origine est-européenne. Mais le débat est vicié à plusieurs égards. En premier lieu par le bilan négatif de cette mesure de protection des salariés est-allemands, adoptée expressément à l'origine en dérogation au droit allemand de la régulation sociale pour répondre à une situation d'urgence : la crise du BTP. Non seulement le salaire minimum n'a pas enrayé la crise du BTP : depuis le milieu des années 1990, le CA de la branche a chuté de $30 \%$
Focalisation sur la Pologne

Sur la sellette en réalité : le travail illégal...

... et les effets pervers de la loi allemande de 1996 sur les travailleurs détachés

\section{Crise du BTP et} travail au noir... 
... mais retour en force du salaire minimum

ver.di et ATTAC orchestrent le chœur des critiques dans l'ensemble de l'Allemagne pour tomber à 95 milliards $€$; quant au nombre d'emplois, il a baissé de $45 \%$ et ne se situe aujourd'hui plus qu'à 770000 . II est vrai que la crise dans les nouveaux Länder a d'autres origines que la concurrence de la main-d'oeuvre est-européenne (ou portugaise) : en particulier des subventions trop massives au secteur dans le cadre de l'Aufbau Ost (voir dans ce numéro), de même qu'une relation inadéquate entre la productivité et le coût salarial. En rehaussant ce coût, le salaire minimum légal dans le BTP $(8,95 €$ par heure à l'est et $10,36 €$ à l'ouest de la RFA dans l'activité principale du BTP) a aussi largement contribué à entretenir la crise du secteur. Soit dit en passant, la faillite médiatisée du groupe Holzmann et, plus récemment, du groupe Walter Bau, n'est certainement pas étrangère à l'attention dont jouit actuellement le BTP. En second lieu, l'introduction de la loi s'est traduite par une forte progression du travail illégal (Européens de l'Est principalement) ; un phénomène qui touche également la gastronomie, la boucherie, l'entretien ou encore la culture maraîchère - les très faibles rémunérations y sont monnaie courante, ce qui explique aussi la difficulté de recruter en toute légalité. Dans le seul BTP, Destatis estime le CA de l'économie parallèle à quelque 35 milliards $€$, un chiffre que l'expert autrichien Friedrich Schneider (voir REA 70/05) porte plus volontiers à près du triple. Le débat se trouve vicié, en troisième lieu, par l'impuissance des autorités nationales à endiguer la pratique abusive de la liberté d'installation des travailleurs indépendants (via la création, dans le pays d'origine, de sociétés qui ne sont que des boîtes aux lettres), qui contribue elle aussi à expliquer la progression du travail au noir. Ces questions requièrent normalement un traitement spécifique de la part de la justice ou du législateur de l'Etat membre concerné.

Or la réponse proposée par le gouvernement allemand pour lutter contre le « dumping salarial » est d'un autre ordre : généraliser le salaire minimum. Cette question, débattue l'an dernier dans le cadre des réformes Hartz, et enterrée à l'automne face à l'opposition des syndicats, resurgit ainsi à l'ombre de la directive 'Services'. Elle se trouve traitée simultanément avec le projet, adopté le 14 avril par le gouvernement et l'opposition, de permettre aux chômeurs de longue durée indemnisés au titre de l'Arbeitslosengeld // créé par la loi Hartz IV (voir REA 68/04) d'accroître leurs revenus complémentaires de quelque $100 €$ de plus (au-delà des « jobs à $1 €$ »). Parallèlement, le secrétaire général du SPD a déclaré la guerre au "capitalisme " le 13 avril dans son discours d'ouverture à l'élaboration du nouveau programme de son parti. L'approche des élections en Rhénanie du Nord-Westphalie amène ainsi le parti du chancelier (que les sondages donnent perdant dans son fief) à afficher un discours social 'de gauche' alors que le chancelier est identifié par la base à une politique plus 'libérale' depuis les lois Hartz. Ce faisant, le parti social-démocrate et la coalition gouvernementale, suivis par l'opposition CDU/CSU sur le dossier du salaire minimum (par surenchère électoraliste plus que par conviction) reprennent à leur compte des positions défendues essentiellement par le syndicat des services ver.di. Son concurrent IG Metall est presque absent du débat, bien que nombre de services aux entreprises relèvent de son domaine de compétences.

C'est en effet ver.di qui mène le camp des opposants syndicaux, s'alliant au groupe ATTAC pour lutter, en Allemagne comme à Bruxelles, contre le « dumping fiscal, social, écologique et sur la qualité » qu'introduirait le projet de directive pour les salariés comme pour les consommateurs. Car ce projet « ne vise qu'un seul objectif: la concurrence. Les objectifs sociaux n'y jouent aucun rôle » affirme l'un de ses tracts diffusés par Internet. Et le syndicat réclame en toute logique une modification du texte ainsi que la suppression du principe du pays d'origine. II n'est certes pas la seule organisation à se montrer insatisfaite de la rédaction du projet qui, de l'avis de nombreux experts et responsables des fédérations professionnelles ou du monde politique allemand et européen, pêche par un certain nombre d'imprécisions. Cela est tellement vrai que le Conseil européen a rédigé une série de «notes explicatives » visant à les lever. 
Mais après tout, c'est là le sort réservé à tout projet de texte de loi, et le débat parlementaire a pour objectif de clarifier certaines questions comme de permettre d'ajuster les diverses positions. Mais ce n'est pas là l'approche affichée par ver.di dont 'l'argumentaire' est caractéristique de la seconde catégorie de positions diffusées dans l'espace public.

Le syndicat, en réalité un conglomérat de métiers (de l'éboueur au chanteur d'opéra en passant par l'employé de la fonction publique ou la vendeuse), est confronté à une énorme disparité de situations. II peine à élaborer une culture unitaire et éprouve de grandes difficultés à organiser le partenariat social au niveau de sa 'branche' (voir REA 59/02). Car le secteur protéiforme des services, dont de larges segments relèvent d'activités nouvellement créées, souvent par externalisation ou essaimage de l'industrie, échappe largement à la norme de branche en matière de temps de travail ou de salaires: le patronat et les salariés ne sont que très faiblement organisés. Par ailleurs, les salaires pratiqués dans la 'branche' relevant du champ de compétences de ver.di sont souvent inférieurs à ceux qui caractérisent l'industrie. La création d'un salaire minimum, outil indirect pour 'organiser' les services et permettre un 'rattrapage' salarial par rapport à l'industrie, est dès lors une revendication classique du syndicat. II bénéficie aujourd'hui, dans le contexte de la crainte du " dumping social » entretenue par l'élargissement européen et instrumentalisée à propos de la directive, d'un contexte électoral favorable permettant d'accroître sa notoriété et son influence. Enfin, à la différence d'IG Metall dont l'identité comme le rôle dans la macro-régulation sociale allemande se fondent sans rupture sur l'héritage du syndicat unitaire de branche, le syndicat ver.di, né en 2001 de la fusion de cinq organisations, est depuis en quête de légitimité. II la cherche, à défaut, en développant une fonction tribunicienne.

Cela étant, la question du salaire minimum est d'une grande complexité. Nombre d'économistes considèrent que non seulement, il ne s'agit pas d'un instrument adapté à la lutte contre le chômage (c'est l'autre problème qui alimente les crispations allemandes), mais qu'il a des effets dommageables pour l'emploi, a fortiori à un moment où l'économie allemande est en quête de flexibilité en matière de temps de travail comme de salaires (voir REA 68/04). Car depuis longtemps, de l'avis unanime des experts, le niveau élevé d'un certain nombre de salaires planchers (minima de branche) constitue l'une des principales 'rigidités' du marché de l'emploi outre-Rhin. Dans ce contexte, l'introduction d'un salaire minimum légal qui, selon les revendications, devrait être établi dans une fourchette allant de $1000 €$ à $1500 €$ mensuels, risque de surcroît de développer le travail au noir comme de renforcer les trappes à inactivité que cherchent justement à supprimer largement les lois Hartz en abaissant le seuil du salaire minimum implicite (voir REA 68/04). S'il n'existe pas de salaire minimum légal outre-Rhin (ni dans six autres Etats membres), ce qu'interdit le principe de l'autonomie des partenaires sociaux inscrit dans la Loi fondamentale, il n'en existe pas moins une multitude de minima de branche qui ont la même fonction. Ils varient généralement selon les régions (même au sein d'un Land) et suivent des régimes différents selon qu'il s'agit d'ouvriers ou d'employés. Ils varient par exemple entre $544 €$ (ouvrier dans la coiffure à Berlin) et $1950 €$ (assurance). Seules quelques rares branches ont adopté des minima valant sur l'ensemble du territoire, dont le secteur de l'assurance (couvert par ver.di).

Théoriquement, rien n'interdit à l'Allemagne de se doter de salaires minima. Si les partenaires sociaux sont unanimes sur le fait qu'il ne peuvent être que variables selon les branches, deux solutions sont possibles: soit la voie légale, soit la voie contractuelle. II existe ainsi une loi de 1952 (Gesetz über Mindestarbeitsbedingungen) permettant d'adopter des conditions de travail minimales pour certains secteurs, mais seulement à condition qu'il n'y ait aucune régulation par les partenaires sociaux. II s'agit en réalité d'une procédure d'ultime recours, et qui n'a jamais été appliquée. Le gouvernement fédéral, SPD en tête, tente aujourd'hui une voie légale larvée via la généralisation de la loi sur les ver.di se cherche une fonction tribunicienne

Un salaire minimum légal ? Il existe de nombreux minima de branche

Salaires minima : voie légale ou voie contractuelle? 
travailleurs détachés. Cela permettrait de contourner la difficulté qui consisterait à accorder force de loi, sur l'ensemble du territoire, aux accords contractuels de branche en vertu d'un article de la Loi sur la convention tarifaire de branche (Tarifvertragsgesetz) permettant au ministre fédéral du Travail, en concertation avec les partenaires sociaux, de leur conférer un caractère " d'utilité générale " (Allgemeinverbindlichkeit). Cette déclaration s'assimile à la procédure française d'extension des conventions collectives. Or le patronat, et même une partie des syndicats, y est opposé. Car l'autre solution - que privilégient de loin les partenaires sociaux puisqu'il en va de leur statut et fonction dans la macro-régulation sociale allemande - est de préserver la voie contractuelle de branche...

LE VRAI DEFI QUE SOULEVE LE PROJET DE DIRECTIVE, et qui nourrit critiques et fantasmes, réside dans ce qu'il révèle un modèle social, allemand ou européen, dont les modes de régulation sont aujourd'hui en porte-à-faux avec les lois régissant l'économie et les profondes mutations qu'ont connues les activités au cours des trente dernières années. L'insuffisant recours à la méthode de l'harmonisation et l'absence d'une liste détaillée des services (cette " liste positive » que réclament certains) servent alors de prétexte pour incriminer "Bruxelles » et un projet, pourtant conjoint, mais qui implique de profonds choix de société.

Car la particularité de l'achèvement du marché intérieur, au plan de la libre circulation des services, est qu'il force indirectement à poser la question du délicat équilibre entre les choix nationaux en matière de protection sociale comme de régulation du travail - qui relèvent de la prérogative des Etats membres et, dans le cas de l'Allemagne plus nettement encore qu'à l'échelon de l'UE, du dialogue social entre partenaires sociaux souverains - et la poursuite de l'intégration de l'espace européen. "Pour promouvoir la croissance et l'emploi et pour renforcer la compétitivité, le marché intérieur des services doit être pleinement opérationnel tout en préservant le modèle social européen. A la lumière du débat en cours, qui montre que la rédaction actuelle de la proposition de directive ne répond pas pleinement aux exigences, le Conseil européen demande que tous les efforts soient entrepris dans le cadre du processus législatif pour dégager un large consensus répondant à l'ensemble de ces objectifs ». Voilà comment les conclusions de la présidence du Conseil européen des 22 et 23 mars 2005 (art. 22, al. 2) traduisent en langage diplomatique la difficulté des choix qui se résume à une simple question. Quel modèle social européen voulons-nous : un modèle social où prédomine l'interventionnisme réglementaire indifférencié ou la régulation sectorielle contractuelle?

\section{Indications bibliographiques}

Comite economique et social europeen, Avis du CESE sur le thème « Pour une meilleure mise en œuvre de la stratégie de Lisbonne », Bruxelles, 27-10-2004 [ECO/153 CESE 1438/2004]

Commission des Communautes europeennes, Proposition de Directive du Parlement européen et du Conseil relative aux services dans le marché intérieur [SEC (2004) 21] en date du 13-01-2004, COM(2004) 2 final

COMmission des CommunaUtes eUROPEenNeS, L'état du marché intérieur des services. Rapport de la Commission au Conseil et au Parlement européen. Rapport présenté dans le cadre de la première phase de la stratégie pour le marché intérieur des services, Bruxelles, 30-07-2002 [COM(2002) 441 final]

CONSEIL DE L'UNION EUROPEENNE, Conseil européen de Bruxelles 22 et 23 mars 2005, Conclusions de la Présidence (7619/05)

PaRlement europeen, Commission du marché intérieur et de la protection des consommateurs, Document de travail sur la proposition de directive du Parlement européen et du Conseil relative aux services dans le marché intérieur (COM(2004)0002) du 13 janvier 2004, en date du 21-12-2004 (PE 353.297)

Relever le défi. La stratégie de Lisbonne pour la croissance et l'emploi, Rapport du groupe de haut niveau présidé par M. Wim Kok, novembre 2004 